\title{
Diabetic Peripheral Neuropathy Associated with Cardiovascular Risk Factors and Glucagon-Like Peptide-I Concentrations Among Newly Diagnosed Patients with Type 2 Diabetes Mellitus
}

\begin{abstract}
Tuan Dinh Le, (iD) 1,2,* Nga Phi Thi Nguyen, ${ }^{2}$ Hoa Thanh Thi Tran, $\left(\mathbb{D}^{3}\right.$

Thuc Luong Cong, ${ }^{4} *$ Lan Ho Thi Nguyen, ${ }^{5}$ Binh Do Nhu, ${ }^{6}$ Son Tien Nguyen, ID $^{2}$ Manh Van Ngo, ${ }^{7}$ Hoa Trung Dinh, (iD) 8 Hien Thi Nguyen, ${ }^{9}$ Kien Trung Nguyen, (ID 10 Duc-Cuong Le (iD) 7,11 *

'Center of Emergency, Critical Care Medicine and Clinical Toxicology, Military Hospital 103, Vietnam Military Medical University, $\mathrm{Ha} \mathrm{Noi}$, Vietnam; ${ }^{2}$ Department of Rheumatology and Endocrinology, Military Hospital 103, Vietnam Military Medical University, $\mathrm{Ha} \mathrm{Noi}$, Vietnam; ${ }^{3}$ National Hospital of Endocrinology, Hanoi, Vietnam; ${ }^{4}$ Department of

Cardiology, Military Hospital 103, Vietnam Military Medical University, Hanoi, Vietnam; ${ }^{5}$ Department of General Internal Medicine, National Hospital of Endocrinology, Ha Noi, Vietnam; ${ }^{6}$ Division of Military Science, Military Hospital I03, Vietnam Military Medical University, Ha Noi, Vietnam; ${ }^{7}$ Postgraduate Training Management Department, Thai Binh University of Medicine and Pharmacy, Thai Binh, Vietnam; ${ }^{8}$ Department of Requested Treatment, National Hospital of Endocrinology, $\mathrm{Ha}$ Noi, Vietnam; ${ }^{9}$ Department of Physiology, Thai Binh University of Medicine and Pharmacy, Thai Binh, Vietnam; ${ }^{10}$ Department of Science Management, Thai Binh University of Medicine and Pharmacy, Thai Binh, Vietnam; "'EpidemiologyFaculty of Public Health, Thai Binh University of Medicine and Pharmacy, Thai Binh, Vietnam
\end{abstract}

*These authors contributed equally to this work

Correspondence: Tuan Dinh Le

Center of Emergency, Critical Care Medicine and Clinical Toxicology, Department of Rheumatology and Endocrinology, Military Hospital 103, Vietnam Military Medical University, 26I Phung Hung Street, Phuc La Ward, Ha Dong District, Ha Noi, Vietnam $\mathrm{Tel}+840388166078$

Email letuan985@gmail.com

Duc-Cuong Le

Postgraduate Training Management Department: Epidemiology-Faculty of Public Health, Thai Binh University of Medicine and Pharmacy, 373 Ly Bon Street, Ky Ba Ward, Thai Binh, Vietnam

Tel +84938042668

Email cuongldvn@gmail.com
Purpose: This study was aimed at the prevalence, cardiovascular risk factors of diabetic peripheral neuropathy (DPN), and the relationship between DPN and fasting glucagon-like peptide-1 (fGLP-1) concentrations in newly diagnosed patients with type 2 diabetes mellitus (nT2D).

Methods: A cross-sectional descriptive study was conducted from 2015 to 2020 with a population of $473 \mathrm{nT} 2 \mathrm{D}$. Screening for DPN was based on the United Kingdom screening test. fGLP-1 was measured by enzyme-linked immunosorbent assay.

Results: The prevalence of DPN was $26.6 \%$, in which mild grade was $17.3 \%$, moderate grade was $8.2 \%$ and severe grade was $1.1 \%$ in total. Age $(\mathrm{OR}=1.73,95 \% \mathrm{CI} 1.12-2.67, \mathrm{p}=0.012)$, smoking $(\mathrm{OR}=1.64,95 \%$ CI 1.03-2.62, $\mathrm{p}=0.037)$, poor control HbAlc $(\mathrm{OR}=2.66,95 \% \mathrm{CI} 1.23-5.76$, $\mathrm{p}=0.01), 24-\mathrm{h}$ urinary albumin $(24 \mathrm{hUA})(\mathrm{OR}=2.49,95 \% \mathrm{CI} 1.26-4.94, \mathrm{p}=0.007)$, and diabetic retinopathy $(\mathrm{OR}=3.17,95 \% \mathrm{CI} 1.46-6.89, \mathrm{p}=0.002)$ significantly increased the risk for DPN. In multivariate logistic regression analysis, hypertension $(\mathrm{OR}=2.96,95 \% \mathrm{CI} 1.16-7.55, \mathrm{p}=0.023)$, triglyceride $(\mathrm{OR}=1.50,95 \% \mathrm{CI} 1.11-2.03, \mathrm{p}=0.009)$, albumin $(\mathrm{OR}=0.85,95 \%$ CI $0.75-0.95$, $\mathrm{p}=0.005)$, and fGLP-1 (OR $=0.79,95 \%$ CI $0.67-0.93, \mathrm{p}=0.005)$ correlated with DPN. The fGLP-1 concentrations were reduced significantly in DPN $(\mathrm{p}<0.001)$. In particular, male patients with DPN had a significantly lower fGLP-1 levels than those without DPN ( $p<0.001)$.

Conclusion: The prevalence of DPN among nT2D was $26.6 \%$. Age, smoking, hypertension, $\mathrm{HbA} 1 \mathrm{c}$ control, triglyceride, albumin, 24hUA, diabetic retinopathy were the associated risk factors of DPN, and fGLP-1 was negatively correlated with DPN (OR $=0.79,95 \%$ CI $0.67-$ $0.93, \mathrm{p}=0.005)$.

Keywords: newly diagnosed type 2 diabetes mellitus, diabetic peripheral neuropathy, cardiovascular risk factors, glucagon-like peptide-1 concentrations

\section{Introduction}

Diabetic peripheral neuropathy (DPN) is the most common complication of diabetes in general and occurs early. ${ }^{1-3}$ Diabetic neuropathy is a type of nerve damage all over the body and can alter autonomic motor, and sensory functions. ${ }^{4} \mathrm{DPN}$ is the most common form of diabetic neuropathy, affecting the outer nerves of the limbs. It directly leads to changes in sensory function, abnormal feelings, and progressive numbness, and increases the risk of foot ulceration, amputation, cognitive dysfunction, and economic burden. ${ }^{5,6}$ Previous studies show that the prevalence of DPN 
among patients with type 2 diabetes accounts for 2.0 $78.8 \%$, depending on the country, duration of diabetes, and examination methods. ${ }^{1,7-9}$ Nerve damage not only leads to loss of feeling that allows injuries to go unnoticed and expandable but also causes serious infections and possible amputations. The risk of amputation in diabetes patients may be more than 25 times greater than that of the non-diabetic population. ${ }^{10}$

The main mechanisms involved in peripheral nerve damage in type 2 diabetes are hyperglycemia, dyslipidemia, and insulin resistance. Firstly, hyperglycemia leads to mitochondrial dysfunction, endoplasmic reticulum stress, promotion of reactive oxygen generation, stimulation the sorbitol accumulation, and advanced glycation end products. $^{8,11,12}$ Secondly, dyslipidemia increases free fatty acid-induced lipotoxicity, reactive oxidative stress, inflammation, cytokine, and chemokine production. ${ }^{12,13}$ Thirdly, insulin resistance develops peripheral neuropathy via reactive oxidative stress and inflammation. ${ }^{14,15}$

Recently, studies have demonstrated that GLP-1 plays in the mechanism of DPN. GLP-1 may have direct nutritional activities on the nervous system, increase neurite outgrowth of adult sensory neurons in vitro like nerve growth factor, ${ }^{16,17}$ and play a role as a neuroprotective agent. ${ }^{18,19}$ Besides, GLP-1 may defend against pyridoxineinduced sensory neuropathy, which shows that GLP-1 could be a potential treatment in diabetic neuropathies. ${ }^{20}$ Furthermore, GLP-1 has been proved to have benefits in microvascular complication diabetes - one mechanism leading to DNP, such as decreasing endothelium dysfunction, which is an early marker of atherosclerosis, arterial endothelial wall injury, and decrease peripheral nerve nutrition. $^{21,22}$

In Vietnam, the prevalence of diabetes is increasing rapidly (accounted for 5.0 to $6 \%$ of the total population). Chronic complications of diabetes are growingcomplex and burdening the healthcare system. DPN appears early at the time of diagnosing diabetes, causes difficulties in treatment, increases the rate of foot complications, amputation, and reduces thequality of life of patients. There is very little documentation about the DPN in nT2D in Viet Nam, and the correlation between plasma GLP-1 concentrations and DPN has not been elucidated. We carried out this study to estimate the prevalence, cardiovascular risk factors of DPN, and the association between DPN with GLP-1 concentrations among newly diagnosed patients with type 2 diabetes in Viet Nam.

\section{Materials and Methods}

\section{Study Population and Design}

A cross-sectional descriptive study was implemented in 473 Vietnamese patients with nT2D in the National Endocrinology Hospital (Hanoi, Vietnam) from January 2015 to December 2020.

Regarding type 2 diabetes, the diagnosis was based on the American Diabetes Association guideline (2019) ${ }^{23}$ for the first time and patients did not relate to any antihyperglycemic treatments (such as biguanides, sulfonylureas, glitazones, incretin therapy, alpha-glucosidase inhibitor, sodium-glucose cotransporter-2 inhibitors, insulin therapy, etc). We excluded patients with conditions that affected GLP-1 concentrations (such as gastrointestinal surgery, bleeding ulcerative colitis, enteritis), and patients with severe heart failure, hemodynamic diseases (such as hemolytic anemia, aplastic anemia, moderate-to-severe anemia), systemic diseases (scleroderma, systemic lupus erythematosus), and severe diabetic conditions (hyperglycemic crises such as hyperosmolarity and diabetic ketoacidosis, hypoglycemia), amputation. Peripheral neuropathy was due to other conditions such as infections (HIV, tuberculosis, severe other infections), autoimmune diseases, inherited disorders, tumors, bone marrow disorders, end-stage renal disease, liver disease, hypothyroidism.

\section{Clinical and Biochemical Assessments}

General information of all patients was collected according to a uniform record. The patients were asked about age; gender; history of drug use, other comorbidities; smoking (tobacco); drinking (beer or wine); anthropometric measurements (height, weight, waist and hip circumference, waist-hip ratio, blood pressure, and pulse). Thorough clinical examination for symptoms of DPN at feet, legs, hands, and arms such as numbness, the reduction of ability to feel pain or temperature changes, tingling or burning sensation, sharp pains or cramps, increased sensitivity to touch, foot problems (ulcers, infections, and bone and joint pain) ${ }^{13,24}$ were performed.

Fasting blood samples were collected from 6:30 to 7:00 the next morning (the patients were asked to fast for at least 8 hours). Fasting plasma glucose (FPG) concentration was quantified by using a hexokinase assay (Beckman AU680, USA). Measurements of HbA1c were carried out by the high-performance liquid chromatography method (Adams A1C, Japan). Total cholesterol, triglyceride, LDL$\mathrm{C}, \mathrm{HDL}-\mathrm{C}$, albumin, aspartate and alanine aminotransferase, creatinine, electrolytes, and hsCRP were measured 
quantitatively by the enzyme colorimetric method (Beckman AU680, USA). Blood insulin and C-peptide concentrations were measured by electrode chemiluminescence (COBAS E411, USA). Quantification of albumin, 24-hour urine was measured by auto-analyzed and immunoturbidimetric method (Beckman AU680, USA). White blood cell was counted by K-4500, Japan. Beta-cell function (HOMA-B) and insulin sensitivity (HOMA-S) and insulin resistance (HOMA-IR) were calculated from the automatic calculator available from https://www.dtu.ox.ac. uk/homacalculator/ (Diabetes Trial Unit, University of Oxford, United Kingdom).

The criteria used in our study were as follows: for hypertension: systolic blood pressure (SBD) $\geq 140 \mathrm{mmHg}$ and/or diastolic blood pressure (DBP) $\geq 90 \mathrm{mmHg}$ at least $2-3$ visits with 2-3 measurements per visit; for glucose control grade: good-normal $\mathrm{FPG}<7.0 \mathrm{mmol} / \mathrm{L}$, poor control: $\mathrm{FPG} \geq 7.0$ $\mathrm{mmol} / \mathrm{L}$; for $\mathrm{HbAlc}$ control grades: good-normal control: $\mathrm{HbAlc}<6.5 \%$, poor control: HbAlc $\geq 6.5 \%$; for lipid disorders: if patients have at least one of the disorders namely high total cholesterol, high triglycerides, high LDL-C, and low HDL-C; for diabetic retinopathy: based on ophthalmoscopy, with proliferative or pre-proliferative retinopathies being diagnosed by an ophthalmologist.

\section{Quantification of GLP-I Levels by Enzyme-Linked Immunosorbent Assay (ELISA)}

Fasting blood samples were collected from patients for fGLP-1 analysis at the same time as for other biochemical assessments. Collecting tubes were added dipeptidyl peptidase-4 inhibitor (DPP-IV Inhibitor, K579, BioVision, USA) before adding blood to avoid inactivation of GLP1 by DPP-4. Then, these samples were centrifuged at $3000 \mathrm{rpm}$ for 15 minutes to get the plasma. Finally, the plasma was analyzed with commercially available kits (Human ELISA GLP-1 kits, IBL, Japan, \#27784) according to the manufacturer's protocol by ELISA apparatus (ThermoFisher Scientific, USA). Color changes were checked at a wavelength of $450 \mathrm{~nm}$. The intra-assay and inter-assay coefficients of variation were less than $10 \%$ in enzyme immunoassays.

\section{Screening for Peripheral Neuropathy}

Screening for DPN was performed based on the United Kingdom screening test including symptom score and grade. $^{25}$
Symptoms: 1) Abnormal sensations felt: burning, numbness or tingling: 2 points; fatigue, aching or cramping: 1 point. 2) Site of discomfort: feet or soles: 2 points; calves: 1 point; elsewhere: 0 points. 3) Time of worst symptoms: night only: 2 points; both day and night: 1 point; day only/do not know: 0 points. 4) Alleviating factor: walking around: 2 points; standing: 1 point; sitting, lying, or no relief: 0 points. 5) Night-time awakening: Yes: 1 point; No: 0 points.

Grade: 0-2 points: (Normal) No peripheral neuropathy 3-4 points: Mild peripheral neuropathy

5-6 points: Moderate peripheral neuropathy

$7-9$ points: Severe peripheral neuropathy

\section{Ethical Statement}

All patients were provided written informed consents and agreed to participate in this study. The protocol was approved by the Institutional Review Board of Vietnam Military Medical University, Vietnam (Reference No 1996/2014/ IRB-VMMU). The study was also conducted using good clinical practice following the Declaration of Helsinki.

\section{Statistical Analysis}

All variables were checked for normality with Kolmogorov Smirnov test if sample size $>50$ or Shapiro-Wilk test if $n<50$. Continuous variables (such as age, BMI, HbAlc, total cholesterol, HDL-C, LDL-C, fGLP-1, etc) were expressed as mean \pm standard deviation for normal distribution variables or median (interquartile range, Q1-Q3) for skewed distribution variables. Differences between groups were examined with either Student's $t$-test or Mann-Whitney test. Categorical variables (such as hypertension, gender, DPN, smoking, drinking, etc) were expressed as percentage. The chi-square test for association was used to determine if there was a relationship between two categorical variables. The relationship between fGLP-1 concentrations and DPN was estimated using the MannWhitney test. Multivariate logistic regression analyses were chosen upon evaluation of the effects of fGLP- 1 concentrations and other factors and DPN. Two-tailed $P$-values $<0.05$ were set as statistically significant.

Data were analyzed with the SPSS version 26 (64-bit edition) for Windows (SPSS Inc, Chicago, IL).

\section{Results}

\section{Demographic and Biomedical Findings}

Age, fasting insulin levels, HDL-C, sodium ion, and HOMA$\mathrm{B}$ in women were significantly higher than those in men. Male patients had higher waist and hip circumference, waist-to-hip 
ratio, smoking, drinking, fasting blood glucose, HbAlc, aspartate aminotransferase, creatinine than those in women. There was no significant difference in both SBP, C-peptide, albumin, total cholesterol, triglyceride, LDL-C, alanine aminotransferase, fGLP-1, hsCRP, white blood cells, potassium ion, 24hUA, HOMA-S, HOMA-IR between male and female patients. Regarding the characteristics of diabetes, albeit those patients were firstly diagnosed with type 2 diabetes, their FPG, HbA1c, and HOMA-IR indices values were high, but BMI, HOMA-S, and HOMA-B indices values were low (Table 1).

\section{Prevalence of Peripheral Neuropathy in Newly Diagnosed Patients with Type 2 Diabetes}

In this study, the prevalence of DPN was $26.6 \%$ (in which, male patients made up $52.3 \%$ and female patients made up $47.7 \%$ ). Regarding the severity of DPN, the percentage for mild DPN, moderate DPN, and severe DPN were $17.3 \%$, $8.2 \%$, and $1.1 \%$, respectively, in total. There was no statistically significant association between gender and DPN $(\mathrm{p}=0.966)$ (Table 2).

Table I The Demographic and Biomedical Characteristics of the Study Population

\begin{tabular}{|c|c|c|c|}
\hline Variables, Unit & $\begin{array}{c}\text { Men } \\
(n=247)\end{array}$ & $\begin{array}{l}\text { Women } \\
(n=226)\end{array}$ & $\begin{array}{c}\text { Overall } \\
(n=473)\end{array}$ \\
\hline Age, years & $52.00(44.00-59.00)^{++}$ & $55.00(50.00-62.00)^{++}$ & $54.00(47.00-60.00)$ \\
\hline BMI, $\mathrm{kg} / \mathrm{m}^{2}$ & $22.40(20.80-24.24)$ & $22.00(20.11-24.20)$ & $22.12(20.3 \mid-24.21)$ \\
\hline Waist circumference, $\mathrm{cm}$ & $84.84 \pm 8.00^{++}$ & $81.16 \pm 8.45^{++}$ & $83.30 \pm 8.35$ \\
\hline Hip circumference, $\mathrm{cm}$ & $93.00(89.00-97.00)^{+}$ & $90.00(86.00-95.00)^{+}$ & $92.00(87.00-96.50)$ \\
\hline Waist-hip radito & $0.91(0.88-0.94)^{++}$ & $0.89(0.86-0.93)^{++}$ & $0.90(0.87-0.94)$ \\
\hline Smoking & $103(47.1)^{++}$ & I $(0.4)^{++}$ & $104(22.0)$ \\
\hline Drinking & $162(65.6)^{++}$ & $5(2.2)^{++}$ & $167(35.3)$ \\
\hline SBP, mmHg & $123.00(\mid 20.00-140.00)$ & $\mid 20.00(|| 4.50-\mid 40.00)$ & $120.00(120.00-140.00)$ \\
\hline $\mathrm{DBP}, \mathrm{mmHg}$ & $80.00(70.00-80.00)^{+}$ & $80.00(70.00-80.00)^{+}$ & $80.00(70.00-80.00)$ \\
\hline FPG, $\mathrm{mmol} / \mathrm{L}$ & $9.90(7.50-11.70)^{+}$ & $9.85(7.47-14.3)^{+}$ & $10.70(7.80-15.25)$ \\
\hline HbAlc, \% & $11.80(8.10-15.60)^{+}$ & $8.91(7.08-11.40)^{+}$ & $9.50(7.20-11.40)$ \\
\hline C-peptide, nmol/L & $0.74(0.48-1.01)$ & $0.76(0.54-\mid .20)$ & $0.75(0.50-1.15)$ \\
\hline Insulin, pmol/L & $61.44(38.05-95.50)^{+}$ & $70.86(45.60-106.77)^{+}$ & $65.64(41.64-104.83)$ \\
\hline Albumin, g/L & $41.15(38.55-44.00)$ & 41.00 (39.00-44.00) & 41.10 (39.00-44.00) \\
\hline Triglycerides, mmol/L & $2.16(1.44-3.65)$ & $2.00(1.36-3.28)$ & $2.09(1.40-3.40)$ \\
\hline Total cholesterol, $\mathrm{mmol} / \mathrm{L}$ & $5.3(4.36-6.16)$ & $5.3(4.5 I-6.20)$ & $5.30(4.4 I-6.17)$ \\
\hline $\mathrm{HDL}-\mathrm{C}, \mathrm{mmol} / \mathrm{L}$ & $1.17(0.99-1.43)^{+}$ & $1.24(1.07-1.50)^{+}$ & $1.19(1.02-1.48)$ \\
\hline LDL-C, mmol/L & $3.07(2.45-4.08)$ & $3.12(2.40-4.02)$ & $3.10(2.43-4.05)$ \\
\hline Lipid disorders, positive & $211(85.8)$ & $182(80.8)$ & $393(83.3)$ \\
\hline Alanine transferase, $\mathrm{U} / \mathrm{L}$ & $27.00(21.00-38.00)$ & $26.00(20.00-35.00)$ & $27.00(21.00-36.00)$ \\
\hline Aspartate transferase, U/L & $34.00(24.00-52.00)^{++}$ & $29.00(20.75-44.00)^{++}$ & $31.00(22.00-49.00)$ \\
\hline Creatinine, $\mu \mathrm{mol} / \mathrm{L}$ & $84.00(75.00-92.00)^{++}$ & $69.00(62.00-76.00)^{++}$ & $76.00(67.00-86.00)$ \\
\hline fGLP-I, pmol/L & $6.25(4.46-9.7 I)$ & $6.49(5.20-9.00)$ & $6.37(4.8 I-9.13)$ \\
\hline hsCRP, mg/L & $2.10(1.01-5.22)$ & $2.04(1.01-4.56)$ & $2.10(1.01-4.79)$ \\
\hline Potassium ion, $\mathrm{mmol} / \mathrm{L}$ & $3.90(3.70-4.10)$ & $3.80(3.70-4.00)$ & $3.90(3.70-4.00)$ \\
\hline Sodium ion, $\mathrm{mmol} / \mathrm{L}$ & $137.00(135.00-139.00)^{++}$ & $138.00(136.00-140.00)^{++}$ & $137.00(136.00-139.00)$ \\
\hline White blood cells (G/L) & $7.88(6.52-9.50)$ & $7.50(6.40-9.10)$ & $7.72(6.50-9.27)$ \\
\hline 24hUA, mg & $3.98(0.00-13.09)$ & $3.04(0.00-8.48)$ & $3.61(0.00-11.50)$ \\
\hline 24hUA, ( $\geq 30 \mathrm{mg}$ ) & $21(9.1)$ & $16(7.3)$ & $37(8.2)$ \\
\hline HOMA-S (\%) & $43.40(28.25-62.03)$ & $42.90(26.50-66.00)$ & $43.20(27.15-63.70)$ \\
\hline HOMA-B (\%) & $32.35(16.80-62.10)^{+}$ & $42.40(23.90-78.00)^{+}$ & $36.20(20.20-69.00)$ \\
\hline HOMA-IR & $2.30(1.61-3.58)$ & $2.33(1.54-3.77)$ & 2.31 (1.57-3.70) \\
\hline Diabetic retinopathy & $15(6.6)$ & $13(6.2)$ & $28(6.4)$ \\
\hline
\end{tabular}

Notes: Data were expressed as mean \pm SD or median and interquartile range (QI-Q3); ${ }^{+} \mathrm{P}<0.05 ;{ }^{++} \mathrm{P}<0.00 \mathrm{I}$ (between male and female group).

Abbreviations: BMI, body mass index; SBP, systolic blood pressure; DBP, diastolic blood pressure; FPG, fasting plasma glucose; HDL-C, high-density lipoprotein cholesterol; LDL-C, low-density lipoprotein cholesterol; fGLP-I, fasting glucagon-like peptide-I; hs-CRP, high-sensitivity C-reactive protein; 24hUA, 24-h urinary albumin; HOMA-S, insulin sensitivity; HOMA-B, beta-cell function; HOMA-IR insulin resistance. 
Table 2 The Prevalence and Grade of Diabetic Peripheral Neuropathy in the Newly Diagnosed Patients with Type 2 Diabetes Mellitus

\begin{tabular}{|c|c|c|c|c|}
\hline \multicolumn{2}{|l|}{ Variables } & \multirow{3}{*}{$\begin{array}{c}\text { Men } \\
(n=247)\end{array}$} & \multirow{3}{*}{$\begin{array}{l}\text { Women } \\
(n=226)\end{array}$} & \multirow{3}{*}{$\begin{array}{l}\text { Overall } \\
(n=473)\end{array}$} \\
\hline Peripheral Diabetic & & & & \\
\hline Neuropathy & & & & \\
\hline \multirow{8}{*}{ Grade } & Positive & $66(14.0)$ & $60(12.6)$ & $126(26.6)$ \\
\hline & Negative & $181(38.3)$ & $166(35.1)$ & $347(73.4)$ \\
\hline & $\mathrm{P}^{*}$-Value & \multicolumn{2}{|c|}{$p=0.966$} & \\
\hline & & & & \\
\hline & Mild & $44(9.3)$ & $38(8.0)$ & $82(17.3)$ \\
\hline & Moderate & $18(3.8)$ & $21(4.4)$ & $39(8.2)$ \\
\hline & Severe & $4(0.8)$ & $\mathrm{I}(0.3)$ & $5(1.1)$ \\
\hline & $\mathrm{p}^{*}$-Value & \multicolumn{2}{|c|}{$P=0.534$} & \\
\hline
\end{tabular}

Note: $*$ Chi-square tests; p-value (between men and women).
Cardiovascular Risk Factors of Peripheral Neuropathy in Newly Diagnosed Patients with Type 2 Diabetes

The prevalence of DPN appeared statistically significantly higher in patients aged $\geq 60(34.5 \%)$; smoking (34.6\%), poor HbA1c control (28.6\%), present $24 \mathrm{hUA}$ $(45.9 \%)$ and present diabetic retinopathy (50\%). Age, smoking, poor control HbAlc; present 24hUA, present diabetic retinopathy increased the risk of DPN (OR = 1.73, 95\% CI 1.12-2.67, $\mathrm{p}=0.012$; OR $=1.64,95 \% \mathrm{CI}$ $1.03-2.62, \mathrm{p}=0.037 ; \mathrm{OR}=2.66,95 \%$ CI $1.23-5.76$, $\mathrm{p}=0.01 ; \mathrm{OR}=2.49,95 \%$ CI $1.26-4.94), \mathrm{p}=0.007$, and $\mathrm{OR}=3.17,95 \%$ CI $1.46-6.89, \mathrm{p}=0.002$, respectively. The rate of DPN was not statistically significant compared with the gender, drinking, hypertension, BMI,

Table 3 The Relationship Between Cardiovascular Risk Factors and Diabetic Peripheral Neuropathy in the Newly Diagnosed Patients with Type 2 Diabetes Mellitus

\begin{tabular}{|c|c|c|c|c|}
\hline \multicolumn{2}{|l|}{ Variables, Unit } & \multicolumn{3}{|c|}{ Peripheral Diabetic Neuropathy } \\
\hline & & Positive & Negative & P*; OR, $95 \% \mathrm{Cl}$ \\
\hline Age, years & $\begin{array}{l}<60(n=334) \\
\geq 60(n=139)\end{array}$ & $\begin{array}{l}78(24.4) \\
48(34.5)\end{array}$ & $\begin{array}{l}256(76.6) \\
91(65.5)\end{array}$ & $\begin{array}{c}P=0.012 \\
O R=1.73 \\
(1.12-2.67)\end{array}$ \\
\hline Smoking & $\begin{array}{l}\text { Active }(n=104) \\
\text { None }(n=369)\end{array}$ & $\begin{array}{l}36(34.6) \\
90(24.4)\end{array}$ & $\begin{array}{l}68(65.4) \\
279(75.6)\end{array}$ & $\begin{array}{c}P=0.037 \\
O R=1.64 \\
(1.03-2.62)\end{array}$ \\
\hline Drinking & $\begin{array}{l}\text { Active }(n=167) \\
\text { None }(n=306)\end{array}$ & $\begin{array}{l}47(28.1) \\
79(25.8)\end{array}$ & $\begin{array}{l}120(71.9) \\
227(74.2)\end{array}$ & $p=0.584$ \\
\hline Hypertension & $\begin{array}{l}\text { Positive }(n=141) \\
\text { Negative }(n=332)\end{array}$ & $\begin{array}{l}44(3 \mid .2) \\
82(24.7)\end{array}$ & $\begin{array}{l}97(68.8) \\
250(75.3)\end{array}$ & $p=0.143$ \\
\hline BMI, $\mathrm{kg} / \mathrm{m}^{2}$ & $\begin{array}{l}<23(n=278) \\
\geq 23(n=195)\end{array}$ & $\begin{array}{l}72(25.9) \\
54(27.7)\end{array}$ & $\begin{array}{l}206(74.1) \\
|4|(72.3)\end{array}$ & $p=0.664$ \\
\hline Lipid disorders & $\begin{array}{l}\text { Positive }(n=393) \\
\text { Negative }(n=79)\end{array}$ & $\begin{array}{l}110(28.0) \\
16(20.3)\end{array}$ & $\begin{array}{l}283(72.0) \\
83(79.7)\end{array}$ & $p=0.156$ \\
\hline Glucose control & $\begin{array}{l}\text { Good-normal }(n=7 I) \\
\text { Poor }(n=402)\end{array}$ & $\begin{array}{c}19(26.8) \\
107(26.6)\end{array}$ & $\begin{array}{l}52(73.2) \\
295(73.4)\end{array}$ & $\mathrm{P}=0.98$ \\
\hline $\mathrm{HbAlc}$ control & $\begin{array}{l}\text { Good-normal }(n=6 I) \\
\text { Poor }(n=4 \mid 2)\end{array}$ & $\begin{array}{c}8(13.1) \\
118(28.6)\end{array}$ & $\begin{array}{l}53(86.9) \\
294(71.4)\end{array}$ & $\begin{array}{c}P=0.01 \\
O R=2.66 \\
(1.23-5.76)\end{array}$ \\
\hline 24hUA, mg & $\begin{array}{l}\geq 30(n=37) \\
<30(n=413)\end{array}$ & $\begin{array}{l}17(45.9) \\
105(25.4)\end{array}$ & $\begin{array}{c}20(54.1) \\
308(74.6)\end{array}$ & $\begin{array}{c}P=0.007 \\
O R=2.49 \\
(1.26-4.94)\end{array}$ \\
\hline Diabetic retinopathy & $\begin{array}{l}\text { Positive }(n=28) \\
\text { Negative }(n=409)\end{array}$ & $\begin{array}{l}14(50.0) \\
98(24.0)\end{array}$ & $\begin{array}{c}14(50.0) \\
31 \mid(76.0)\end{array}$ & $\begin{array}{c}P=0.002 \\
O R=3.17 \\
(1.46-6.89)\end{array}$ \\
\hline
\end{tabular}

Note: *Chi-square tests.

Abbreviations: BMI, body mass index; 24hUA, 24-h urinary albumin; OR, odds ratio; Cl, confidence interval. 
lipid disorders and fasting glucose control in univariate analysis $(\mathrm{p}>0.05)$ (Table 3).

\section{Fasting GLP-I Concentrations Correlation to Diabetic Peripheral Neuropathy in Newly Diagnosed Patients with Type 2 Diabetes}

A Mann-Whitney $U$-test showed that there was a statistically significant difference in concentration of fGLP-1 level between different DPN groups $(Z=-3.554 ; \mathrm{p}<0.001)$. There was a statistically significant difference in fGLP-1 between male patients with and without DPN $(Z=-4.103 ; \mathrm{p}<0.001)$. The median fGLP-1 concentration for patients with DPN was $5.27 \mathrm{pmol} / \mathrm{L}$ compared to $6.76 \mathrm{pmol} / \mathrm{L}$ in patients without DPN. Interestingly, while in the male group, the median fGLP-1 for present DPN was $4.11 \mathrm{pmol} / \mathrm{L}$ compared to $6.91 \mathrm{pmol} / \mathrm{L}$ in the non-DPN group, there was no statistically significant difference in fGLP-1 among female patients with and without DPN $(Z=-0.951 ; \mathrm{p}=0.342)$ (Table 4$)$.

The multivariate logistic regression analysis showed that the following factors had correlation to DPN in nT2D: hypertension $(\mathrm{OR}=2.96,95 \%$ CI 1.16-7.55, $\mathrm{p}=0.023), \mathrm{HbA} 1 \mathrm{c}(\mathrm{OR}=1.22,95 \%$ CI $1.01-1.47$, $\mathrm{p}=0.042)$, triglyceride $(\mathrm{OR}=1.50,95 \%$ CI $1.11-2.03$, $\mathrm{p}=0.009)$, albumin $(\mathrm{OR}=0.85,95 \%$ CI $0.75-0.95$,

Table 4 The Relationship Between Fasting GLP-I Concentrations and Diabetic Peripheral Neuropathy in the Newly Diagnosed Patients with Type 2 Diabetes Mellitus

\begin{tabular}{|c|c|c|}
\hline & \multicolumn{2}{|c|}{ Diabetic Peripheral Neuropathy } \\
\hline & Positive & Negative \\
\hline \multicolumn{3}{|c|}{ Mean rank (n) } \\
\hline Male & $28.24(27)$ & $52.90(63)$ \\
\hline Female & $37.22(29)$ & $42.36(5 \mathrm{I})$ \\
\hline Overall & $66.41(56)$ & $94.88(114)$ \\
\hline \multicolumn{3}{|c|}{ Median fGLP-I (pmol/l) (n) } \\
\hline Male & 4.II (27) & $6.91(63)$ \\
\hline Female & $6.37(29)$ & $6.64(5 \mathrm{I})$ \\
\hline Overall & $5.27(56)$ & $6.76(114)$ \\
\hline \multicolumn{3}{|c|}{ Test Statistics** } \\
\hline Male & \multicolumn{2}{|c|}{$Z=-4.103 ; p<0.001, n=90$} \\
\hline Female & \multicolumn{2}{|c|}{$Z=-0.95 I ; p=0.342, n=80$} \\
\hline Overall & \multicolumn{2}{|c|}{$Z=-3.554 ; p<0.001, n=170$} \\
\hline
\end{tabular}

Note: **Mann-Whitney U-tests.

Abbreviation: fGLP-I, fasting glucagon-like peptide-I. $\mathrm{p}=0.005)$, and fGLP- $1(\mathrm{OR}=0.79,95 \%$ CI $0.67-$ $0.93, \mathrm{p}=0.005)($ Table 5).

\section{Discussion}

\section{The Prevalence of Peripheral Diabetic Neuropathy and Risk Factors}

In our study, the prevalence of DPN in nT2D was $26.6 \%$. Regarding the severity of DPN, the percentage for mild DPN, moderate DPN, and severe DPN was $17.3 \%, 8.2 \%$, and $1.1 \%$, respectively, in total. There was no statistically significant association between gender and DPN ( $\mathrm{p}=$ 0.966). Previous studies showed that the prevalence of DPN was different among adults with diabetes depending on the nation, age, duration of diabetes, glucose control, and type 1 versus type 2 diabetes from $9.6 \%$ to $69 \%$ in Latin and American countries ${ }^{1,26,27}$ from $30.1 \%$ to $58 \%$ in Asian countries. ${ }^{28,29}$ Thus, the prevalence of DPN in our study was lower than that in other Asian countries due to several reasons. Firstly, participants in our study were nT2D, while other studies enrolled adult patients with long-duration of diabetes and, perhaps, with a relatively high prevalence of microvascular complications. Secondly, there were differences in methods between studies that were used to screen for DPN. We identified the presence of DPN based on clinical examination (the United Kingdom screening test) and other studies used to $10-\mathrm{g}$ monofilament testing or neurothesiometer testing or electromyography. ${ }^{28}$ Furthermore, the differences in anthropometric characteristics, social and cultural environment of Vietnamese type 2 diabetes compared to other countries such as low BMI (non-obese individuals diabetes), low waist and hip circumference, highly physical activity, or healthy lifestyles, low-fat diet. They were suggested that these factors could delay the onset of chronic complications and metabolic disorders in Vietnamese patients with type 2 diabetes. ${ }^{30,31}$

Studies further demonstrated that risk factors for cardiovascular were predictors of the development of DPN in type 1 and type 2 diabetes patients. ${ }^{32,33}$

In our study, the risk of developing DPN in patients aged $\geq 60$ was higher than that in patients with age $<60$ years $(\mathrm{OR}=1.73, \mathrm{p}=0.012)$. Elderly patients with type 2 diabetes had a higher rate of complications including macro- and micro-vascular disease than young patients. Age was an independent risk factor for the DPN and associated with neurological dysfunction in both major nerve and minor nerve. ${ }^{34,35}$ Besides, previous studies and 
Table 5 The Multivariate Logistic Regression Analysis Associated Other Factors with Diabetic Peripheral Neuropathy in the Newly Diagnosed Patients with Type 2 Diabetes Mellitus

\begin{tabular}{|c|c|c|c|c|}
\hline Determinants, Unit & B & $\mathbf{p}$ & OR & $95 \% \mathrm{Cl}$ \\
\hline Hypertension ( 0 = negative, $\mathrm{I}=$ positive $)$ & 1.084 & 0.023 & 2.96 & $1.16-7.55$ \\
\hline $\mathrm{HbAlc} \%$ & 0.196 & 0.042 & 1.22 & $1.01-1.47$ \\
\hline Triglyceride, $\mathrm{mmol} / \mathrm{L}$ & 0.404 & 0.009 & 1.50 & $1.11-2.03$ \\
\hline Albumin, $g / L$ & -0.166 & 0.005 & 0.85 & $0.75-0.95$ \\
\hline fGLP-I, pmol/L & -0.235 & 0.005 & 0.79 & $0.67-0.93$ \\
\hline HOMA-B, \% & 0.006 & 0.407 & 1.01 & $0.99-1.02$ \\
\hline Urea, $\mathrm{mmol} / \mathrm{L}$ & -0.177 & 0.267 & 0.84 & $0.61-1.15$ \\
\hline Gender $(I=$ male, 2 = female $)$ & 0.585 & 0.185 & 1.79 & $0.76-4.26$ \\
\hline Potassium ion, $\mathrm{mmol} / \mathrm{L}$ & 0.484 & 0.408 & 1.62 & $0.52-5.11$ \\
\hline $\mathrm{HDL}-\mathrm{C}, \mathrm{mmol} / \mathrm{L}$ & 0.794 & 0.110 & 2.21 & $0.84-5.86$ \\
\hline LDL-C, mmol/L & -0.108 & 0.578 & 0.90 & $0.61-1.31$ \\
\hline White blood cells, G/L & -0.068 & 0.430 & 0.93 & $0.79-1.11$ \\
\hline BMI $\left(0=\right.$ BMI $\left.<23, \quad I=B M I \geq 23\left(\mathrm{~kg} / \mathrm{m}^{2}\right)\right)$ & 0.410 & 0.362 & 1.51 & $0.62-3.64$ \\
\hline Glucose control $(0=$ good-normal, $\mathrm{I}=$ poor $)$ & -1.100 & 0.140 & 0.33 & $0.08-1.43$ \\
\hline
\end{tabular}

Abbreviations: fGLP-I, fasting glucagon-like peptide-I; HDL-C, high-density lipoprotein cholesterol; LDL-C, low-density lipoprotein cholesterol; HOMA-B, beta-cell function; BMI, body mass index; OR, odds ratio; $\mathrm{Cl}$, confidence interval.

our study suggested that smoking was the most common risk factor for the development of vascular complications including DPN in type 2 diabetes. ${ }^{36,37}$

Poor HbAlc control increased the risk of DPN in both univariate analysis and multivariate logistic regression analysis in nT2D. Chronic hyperglycemia elevated inflammatory factors that lead to peripheral nerves injuries such as tumor necrosis factor-alpha, interleukin-6,8, monocyte chemoattractant protein-1, transforming growth factor $\beta$, and C-reactive protein, and infiltration of cytokines into the vascular tissue and thus reduces peripheral neuropathy nutrition. ${ }^{38,39}$

In our study, other microvascular complications included $24 \mathrm{hUA} \geq 30 \mathrm{mg}(8.2 \%)$, and diabetic retinopathy $(6.4 \%)$, and these complications were significantly associated with the development of DPN. Recently, studies showed that positive family history, illiteracy, hypertension, higher HbAlc level, and DPN were associated with elevation of cardiovascular diseases events and caused of morbidity and mortality in diabetic patients. ${ }^{2,40,41}$ Diabetic nephropathy is closely related to the expansion of DPN in type 2 diabetes. Urinary albumin excretion rate can be used as predicting DPN process. ${ }^{42-44}$ Changes in urine albumin-to-creatinine ratio ( $\geq 30 \%$ ) increase the risk for new-onset DPN in type 2 diabetes (OR $=3.059,95 \% \mathrm{CI}$ 1.012-9.249). ${ }^{45}$ Furthermore, diabetic retinopathy, especially severe diabetic retinopathy is correlated with DPN; and DPN was a factor like a tell-tale sign of diabetic retinopathy; thus, it is necessary to examine more intensive ophthalmic disease. ${ }^{4}$ All microvascular complications of diabetes arose early, and they were related to each other via complex pathological mechanisms, ${ }^{3,41}$ therefore, it is essential to early detect and manage these complications as soon as possible, which can prevent their development and improve quality of life for type 2 diabetes patients.

\section{The Relation Between Diabetic Peripheral Neuropathy and fGLP-I Concentrations}

Our study outlined the correlation between fGLP-1 concentrations and DPN in n2TD. A Mann-Whitney $U$-test showed that there was a statistically significant difference in fGLP-1 level between the patients with and without DPN. GLP-1 decreased significantly in patients with DPN in male patients and in overall.

Previous studies have reported that GLP-1 secretion was reduced in both fasting and postprandial patients with type 2 diabetes, ${ }^{46-48}$ which resulted in impaired insulin secretion, a decrease in beta-cell function, and an increase in insulin resistance. ${ }^{49,50}$ These effects caused hyperglycemia, oxidative stress, enhancing the synthesis of inflammatory cytokines, tumor necrosis factor (TNF)- $\alpha$ and MCP-1, and microvascular complications. ${ }^{21,51,52}$ GLP-1 influenced DPN via the indirect mechanisms of microvascular damage in type 2 diabetes. Receptor for advanced glycation end-products mRNA transcription was inhibited by GLP-1, leading to reduced reactive oxygen species production and decrease in vascular cell 
adhesion molecule-1 expression. Thus, GLP-1 concentrations contributed to preventing the arterial wall from inflammatory response, atherosclerosis, oxidative stress, and increase endothelium relaxations. ${ }^{22}$ Besides, GLP-1 inhibited the formation of foam macrophages, prevented the inflammatory response to the artery, which was closely related to negative feedback of CD36 and acetyl-coenzyme-A, acetyltransferase1 , and modulates adhesion molecules, such as interleukin-10, CD136, and CD204. ${ }^{53}$ GLP-1 reduced endothelial dysfunction, inflammatory response via the consequence of the decrease in nitrotyrosine, 8 -iso prostaglandin F2 $\alpha$, sICAM-1, and interleukin- $6,{ }^{54}$ which is important to cause neuropathy in type 2 diabetes. ${ }^{9,55}$

Interestingly, in our study, while there was a significant difference between patients with DPN and without DPN in fGLP-1 in male patients, this trend was not seen in female patients. One explanation for this difference between men and women was tobacco use. Tobacco use contributes to the macro- and microvascular complications of type 2 diabetes mellitus. GLP-1 was evidenced to addressing comorbid tobacco use disorder in diabetes with smoking. ${ }^{37}$ GLP-1 or analogs reduced the effect of alcohol to trigger the mesolimbic dopamine system, decreased alcohol intake, and ability selfadministration. ${ }^{56,57}$ In our study, there were very few women who were drinking $(2.2 \%)$ or smoking $(0.4 \%)$ and smoking associated with an increased risk of DPN $(\mathrm{OR}=1.64,95 \% \mathrm{CI}$ $1.03-2.62, \mathrm{p}=0.037$ ). Thus, GLP-1 levels were significantly decreased only in men with DPN, but not in women.

In this study, the multivariate logistic regression analysis showed that the following factors had a significant correlation to DPN in firstly diagnosed type 2 diabetes: hypertension, HbAlc, triglyceride, albumin, and fGLP-1. Particularly, high $\mathrm{HbAlc}$, high triglyceride, low albumin levels, and low fGLP-1 were risk factors for DPN. Previous studies evidenced that decreased levels of serum albumin, elevated triglyceride, and hypertension correlated with progression of DPN. ${ }^{58-60}$

There were some limitations of the study. Our study was conducted on a relatively small sample size. Besides, we had not been able to use electromyography for early diagnosis of peripheral nerve conduction disturbances in patients with type 2 diabetes who had an asymptomatic peripheral neuropathy.

\section{Conclusion}

In conclusion, the prevalence of DPN among nT2D was screened by the United Kingdom screening test was $26.6 \%$. Age, smoking, hypertension, HbAlc control, triglyceride, albumin, 24hUA, diabetic retinopathy were the associated risk factors of DPN. In patients with DPN, GLP-1 concentrations were reduced significantly, and a decrease in GLP-1 was a risk factor for DPN.

\section{Abbreviations}

DPN, diabetic peripheral neuropathy; fGLP-1, fasting glucagon-like peptide-1; nT2D, newly diagnosed type 2 diabetes mellitus; DPP4, dipeptidyl peptidase-IV; ELISA, enzymelinked immunosorbent assay; BMI, body mass index; SD, standard deviation; SBP, systolic blood pressure; DBP, diastolic blood pressure; FPG, fasting plasma glucose; HDL-C, highdensity lipoprotein cholesterol; LDL-C, low-density lipoprotein cholesterol; hs-CRP, high-sensitivity C-reactive protein; 24hUA, 24-h urinary albumin; HOMA, homeostasis model assessment; HOMA-S, insulin sensitivity; HOMA-B, betacell function; HOMA-IR insulin resistance; OR, odds ratio; CI, confidence interval.

\section{Acknowledgments}

We regard to give the best thank you to all the staff in the Outpatients Department of the Vietnam Endocrinology Hospital for supporting the study.

\section{Author Contributions}

All authors made a significant contribution to the work reported, whether that is in the conception, study design, execution, acquisition of data, analysis and interpretation, or in all these areas; took part in drafting, revising or critically reviewing the article; gave final approval of the version to be published; have agreed on the journal to which the article has been submitted; and agree to be accountable for all aspects of the work.

\section{Disclosure}

The authors declare no potential conflicts of interest in this work.

\section{References}

1. Yovera-Aldana M, Velásquez-Rimachi V, Huerta-Rosario A, et al. Prevalence and incidence of diabetic peripheral neuropathy in Latin America and the Caribbean: a systematic review and meta-analysis. PLoS One. 2021;16:e0251642. doi:10.1371/journal.pone.0251642

2. Wani FA, Koul RK, Raina AA, et al.Prevalence of microvascular complications in newly diagnosed type 2 diabetes patients. Int J Curr Res. 2018;10:67231-67233.

3. Dyck PJ, Kratz KM, Karnes JL, et al. The prevalence by staged severity of various types of diabetic neuropathy, retinopathy, and nephropathy in a population-based cohort: the Rochester Diabetic Neuropathy Study. Neurology. 1993;43:817. doi:10.1212/ WNL.43.4.817 
4. Abdollahi A. Neuropathy and retinopathy in diabetes: is there any association? Int $J$ Ophthalmol. 2009;2:57-60.

5. Sothornwit J, Srisawasdi G, Suwannakin A, Sriwijitkamol A. Decreased health-related quality of life in patients with diabetic foot problems. Diabetes Metab Syndr Obes Targets Ther. 2018;11:35.

6. Ni W, Zhang Z, Zhang B, et al. Connecting peripheral to central neuropathy: examination of nerve conduction combined with olfactory tests in patients with type 2 diabetes. Diabetes Metab Syndr Obes Targets Ther. 2021;14:3097-3107. doi:10.2147/DMSO.S312021

7. Kostev K, Jockwig A, Hallwachs A, Rathmann W. Prevalence and risk factors of neuropathy in newly diagnosed type 2 diabetes in primary care practices: a retrospective database analysis in Germany and UK. Prim Care Diabetes. 2014;8:250-255. doi:10.1016/j.pcd.2014.01.011

8. Ko S-H, Cha B-Y. Diabetic peripheral neuropathy in type 2 diabetes mellitus in Korea. Diabetes Metab J. 2012;36:6. doi:10.4093/ dmj.2012.36.1.6

9. Román-Pintos LM, Villegas-Rivera G, Rodríguez-Carrizalez AD, Miranda-Díaz AG, Cardona-Muñoz EG. Diabetic polyneuropathy in type 2 diabetes mellitus: inflammation, oxidative stress, and mitochondrial function. J Diabetes Res. 2016;2016:1-16. doi:10.1155/ 2016/3425617

10. Reiber Ge LJ. Epidemiology and Economic impact of foot ulcers and amputations in people with diabetes. In: Bowker JH, Pfeifer MA, editors. Levin and O'Neal's the Diabetic Foot. Philadelphia, Mosby: Elsevier; 2008:3-32.

11. Fiorentino T, Prioletta A, Zuo P, Folli F. Hyperglycemia-induced oxidative stress and its role in diabetes mellitus related cardiovascular diseases. Curr Pharm Des. 2013;19:5695-5703.

12. Sandireddy R, Yerra VG, Areti A, Komirishetty P, Kumar A. Neuroinflammation and oxidative stress in diabetic neuropathy: futuristic strategies based on these targets. Int $J$ Endocrinol. 2014;2014:1-10. doi:10.1155/2014/674987

13. Feldman EL, Callaghan BC, Pop-Busui R, et al. Diabetic neuropathy. Nat Rev Dis Prim. 2019;5:41. doi:10.1038/s41572-019-0092-1

14. Han L, Ji L, Chang J, et al. Peripheral neuropathy is associated with insulin resistance independent of metabolic syndrome. Diabetol Metab Syndr. 2015;7:14. doi:10.1186/s13098-015-0010-y

15. Hurrle S, Hsu WH. The etiology of oxidative stress in insulin resistance. Biomed J. 2017;40:257-262. doi:10.1016/j.bj.2017.06.007

16. Perry T, Lahiri DK, Chen D, et al. A novel neurotrophic property of glucagon-like peptide 1: a promoter of nerve growth factor-mediated differentiation in PC12 cells. J Pharmacol Exp Ther. 2002;300:958-966.

17. Kan M, Guo G, Singh B, Singh V, Zochodne DW. Glucagon-like peptide 1, insulin, sensory neurons, and diabetic neuropathy. $J$ Neuropathol Exp Neurol. 2012;71:494-510. doi:10.1097/ NEN.0b013e3182580673

18. During MJ, Cao L, Zuzga DS, et al. Glucagon-like peptide-1 receptor is involved in learning and neuroprotection. Nat Med. 2003;9:1173-1179. doi:10.1038/nm919

19. Perry T, Haughey NJ, Mattson MP, Egan JM, Greig NH. Protection and reversal of excitotoxic neuronal damage by glucagon-like peptide-1 and exendin-4. $J$ Pharmacol Exp Ther 2002;302:881-888. doi:10.1124/jpet.102.037481

20. Perry T, Holloway HW, Weerasuriya A, et al. Evidence of GLP-1-mediated neuroprotection in an animal model of pyridoxine-induced peripheral sensory neuropathy. Exp Neurol. 2007;203:293-301. doi:10.1016/j.expneurol.2006.09.028

21. Daisuke YS, Yabe D. Glucose - dependent insulinotropic polypeptide and glucagon - like peptide -1 : incretin actions beyond the pancreas J Diabetes Investig. 2013;4:108-1130. doi:10.1111/jdi.12065

22. Ishibashi Y, Matsui T, Takeuchi M, Yamagishi S. Glucagon-like peptide-1 (GLP-1) inhibits advanced glycation end product (AGE)-induced up-regulation of VCAM-1 mRNA levels in endothelial cells by suppressing AGE receptor (RAGE) expression. Biochem Biophys Res Commun. 2010;391:1405-1408. doi:10.1016/j. bbrc.2009.12.075
23. American Diabetes Association. Standards of medical care in diabetes-2019 abridged for primary care providers. Clin Diabetes. 2019;37:11-34. doi:10.2337/cd18-0105

24. Nascimento OJM, Do Pupe CCB, Cavalcanti EBU. Diabetic neuropathy. Rev Dor. 2016;17. doi:10.5935/1806-0013.20160047

25. Young MJ, Boulton AJM, Macleod AF, Williams DRR, Sonksen PH. A multicentre study of the prevalence of diabetic peripheral neuropathy in the United Kingdom hospital clinic population. Diabetologia. 1993;36:150-154. doi:10.1007/BF00400697

26. Rubino A, Rousculp MD, Davis K, et al. Diagnosis of diabetic peripheral neuropathy among patients with type 1 and type 2 diabetes in France, Italy, Spain, and the United Kingdom. Prim Care Diabetes. 2007;1:129-134. doi:10.1016/j.pcd.2007.07.006

27. Hicks CW, Selvin E. Epidemiology of peripheral neuropathy and lower extremity disease in diabetes. Curr Diab Rep. 2019;19:86. doi:10.1007/s11892-019-1212-8

28. Malik RA, Andag-Silva A, Dejthevaporn C, et al. Diagnosing peripheral neuropathy in South-East Asia: a focus on diabetic neuropathy. J Diabetes Investig. 2020;11:1097-1103. doi:10.1111/jdi.13269

29. Pan Q, Li Q, Deng W, et al. Prevalence of and risk factors for peripheral neuropathy in Chinese patients with diabetes: a multicenter cross-sectional study. Front Endocrinol (Lausanne). 2018;9. doi:10.3389/fendo.2018.00617

30. Thy Khue N. Diabetes in Vietnam. Ann Glob Health. 2016;81:870. doi:10.1016/j.aogh.2016.01.003

31. Nguyen CT, Pham NM, Tran DV, Lee AH, Binns CW. Lifestyle and diet in relation to risk of type 2 diabetes in Vietnam: a hospital-based case-control study. Springerplus. 2016;5:687. doi:10.1186/s40064016-2313-3

32. Tesfaye S, Chaturvedi N, Eaton SEM, et al. Vascular risk factors and diabetic neuropathy. N Engl J Med. 2005;352:341-350. doi:10.1056/ NEJMoa032782

33. Yang C-P, Lin -C-C, Li C-I, et al. Cardiovascular risk factors increase the risks of diabetic peripheral neuropathy in patients with type 2 diabetes mellitus. Medicine (Baltimore). 2015;94:e1783. doi:10.1097/ MD.0000000000001783

34. Mao F, Zhu X, Liu S, et al. Age as an independent risk factor for diabetic peripheral neuropathy in Chinese patients with type 2 diabetes. Aging Dis. 2019;10:592. doi:10.14336/AD.2018.0618

35. Corriere M, Rooparinesingh N, Kalyani RR. Epidemiology of diabetes and diabetes complications in the elderly: an emerging public health burden. Curr Diab Rep. 2013;13:805-813. doi:10.1007/ s11892-013-0425-5

36. Clair C, Cohen MJ, Eichler F, Selby KJ, Rigotti NA. The effect of cigarette smoking on diabetic peripheral neuropathy: a systematic review and meta-analysis. J Gen Intern Med. 2015;30:1193-1203. doi:10.1007/s11606-015-3354-y

37. Mitchell BD, Hawthorne VM, Vinik AI. Cigarette smoking and neuropathy in diabetic patients. Diabetes Care. 1990;13:434-437. doi: $10.2337 /$ diacare.13.4.434

38. Ristikj-Stomnaroska D, Risteska-Nejashmikj V, Papazova M. Role of inflammation in the pathogenesis of diabetic peripheral neuropathy. Open Access Maced J Med Sci. 2019;7:2267-2270. doi:10.3889/ oamjms.2019.646

39. Mussa BM, Srivastava A, Al-Habshi A, et al. Inflammatory biomarkers levels in T2DM Emirati patients with diabetic neuropathy. Diabetes Metab Syndr Obes Targets Ther. 2021;14:3389-3397. doi:10.2147/DMSO.S319863

40. Brownrigg JRW, de Lusignan S, McGovern A, et al. Peripheral neuropathy and the risk of cardiovascular events in type 2 diabetes mellitus. Heart. 2014;100:1837-1843. doi:10.1136/heartjnl-2014305657

41. Ali A, Iqbal F, Taj A, et al. Prevalence of microvascular complications in newly diagnosed patients with type 2 diabetes. Pak J Med Sci. 2013;29:899. 
42. Xu L, Lin X, Guan M, Liu Y. Correlation between different stages of diabetic nephropathy and neuropathy in patients with T2DM: a cross-sectional controlled study. Diabetes Ther. 2018;9:2335-2346. doi:10.1007/s13300-018-0519-9

43. Zhang Y, Jiang Y, Shen X, Yan S. Can both normal and mildly abnormal albuminuria and glomerular filtration rate be a danger signal for diabetic peripheral neuropathy in type 2 diabetes mellitus? NeurolSci. 2017;38:1381-1390.

44. Aleidan FA, Ahmad BA, Alotaibi FA, et al. Prevalence and risk factors for diabetic peripheral neuropathy among Saudi hospitalized diabetic patients: a nested case-control study. Int J Gen Med. 2020;13:881-889.

45. Zhong M, Yang Y-R, Zhang Y-Z, Yan S-J. Change in urine albumin-to-creatinine ratio and risk of diabetic peripheral neuropathy in type 2 diabetes: a retrospective cohort study. Diabetes Metab Syndr Obes Targets Ther. 2021;14:1763-1772.

46. Nauck M, Stöckmann F, Ebert R, Creutzfeldt W. Reduced incretin effect in Type 2 (non-insulin-dependent) diabetes. Diabetologia. 1986;29:46-52.

47. León DD, De Crutchlow MF, Ham J-YN, Stoffers DA. Role of glucagon-like peptide-1 in the pathogenesis and treatment of diabetes mellitus. Int J Biochem Cell Biol. 2006;38:845-859. doi:10.1016/j. biocel.2005.07.011

48. Vilsbøll T, Holst JJ. Incretins, insulin secretion and type 2 diabetes mellitus. Diabetologia. 2004;47:357-366. doi:10.1007/s00125-0041342-6

49. Holst JJ, Gromada J. Role of incretin hormones in the regulation of insulin secretion in diabetic and nondiabetic humans. Am J Physiol Metab. 2004;287:E199-E206.

50. Vilsbøll T. The effects of glucagon-like peptide-1 on the beta cell. Diabetes Obes Metab. 2009;11:11-18. doi:10.1111/j.14631326.2009.01073.x

51. Alonso N, Julián MT, Puig-Domingo M, Vives-Pi M. Incretin hormones as immunomodulators of atherosclerosis. Front Endocrinol (Lausanne). 2012;3. doi:10.3389/fendo.2012.00112
52. Sheikh A. Direct cardiovascular effects of glucagon like peptide-1. Diabetol Metab Syndr. 2013;5:47. doi:10.1186/1758-5996-5-47

53. Shiraishi D, Fujiwara Y, Komohara Y, Mizuta H, Takeya M. Glucagon-like peptide-1 (GLP-1) induces M2 polarization of human macrophages via STAT3 activation. Biochem Biophys Res Commun. 2012;425:304-308. doi:10.1016/j.bbrc.2012.07.086

54. Ceriello A, Novials A, Ortega E, et al. Glucagon-like peptide 1 reduces endothelial dysfunction, inflammation, and oxidative stress induced by both hyperglycemia and hypoglycemia in type 1 diabetes. Diabetes Care. 2013;36:2346-2350. doi:10.2337/dc12-2469

55. Chapouly C, Yao Q, Vandierdonck S, et al. Impaired Hedgehog signalling-induced endothelial dysfunction is sufficient to induce neuropathy: implication in diabetes. Cardiovasc Res. 2016;109:217-227. doi:10.1093/cvr/cvv263

56. Shirazi RH, Dickson SL, Skibicka KP, Caramelli D. Gut peptide GLP-1 and its analogue, exendin-4, decrease alcohol intake and reward. PLoS One. 2013;8:e61965. doi:10.1371/journal. pone.0061965

57. Jerlhag E. GLP-1 signaling and alcohol-mediated behaviors; preclinical and clinical evidence. Neuropharmacology. 2018;136:343-349. doi:10.1016/j.neuropharm.2018.01.013

58. Yan P, Tang Q, Wu Y, et al. Serum albumin was negatively associated with diabetic peripheral neuropathy in Chinese population: a cross-sectional study. Diabetol Metab Syndr. 2021;13:100. doi:10.1186/s13098-021-00718-4

59. Wiggin TD, Sullivan KA, Pop-Busui R, et al. Elevated triglycerides correlate with progression of diabetic neuropathy. Diabetes. 2009;58:1634-1640. doi:10.2337/db08-1771

60. Ponirakis G, Petropoulos IN, Alam U, et al. Hypertension contributes to neuropathy in patients with type 1 diabetes. Am J Hypertens. 2019;32:796-803. doi:10.1093/ajh/hpz058

\section{Publish your work in this journal}

Diabetes, Metabolic Syndrome and Obesity: Targets and Therapy is an international, peer-reviewed open-access journal committed to the rapid publication of the latest laboratory and clinical findings in the fields of diabetes, metabolic syndrome and obesity research. Original research, review, case reports, hypothesis formation, expert opinion and commentaries are all considered for publication. The manuscript management system is completely online and includes a very quick and fair peer-review system, which is all easy to use. Visit http://www.dovepress.com/testimonials.php to read real quotes from published authors. 